\title{
A marker for personalised feedback on dieting: Angiotensin Converting Enzyme
}

\author{
Shilpa Tejpal ${ }^{1}$, Narinder Sanghera ${ }^{1}$, Vijayalaxmi Manoharan ${ }^{1,2}$, Joan Planas-Iglesias ${ }^{1}$ and \\ Judith Klein-Seetharaman ${ }^{1,2}$ \\ ${ }^{1}$ Division of Metabolic and Vascular Health, Medical School, University of Warwick, Gibbet Hill, Coventry, CV4 7AL, UK and \\ ${ }^{2}$ Institute for Digital Healthcare, Warwick Manufacturing Group, University of Warwick, Coventry, CV4 7A, UK
}

Obesity is a worldwide rising epidemic disease and is associated with metabolic disorders such as diabetes and insulin resistance ${ }^{(1)}$. Exercise, drugs, surgery and/or dietary plans are typical weight loss options that are offered/follow to lose weight ${ }^{(2)}$. However, achieving weight loss is hard work and failure is demoralising. Technology supported devices allow to record calorie consumption but relationship no direct link to weight loss ${ }^{(3)}$. There is a need of a devise that could be more directly linked to weight loss. There is first need of identification of a quantitative and science-based biomarker of weight loss that has the potential to provide a biological feedback loop to the dieter. Angiotensin Converting Enzyme (ACE) has been identified as a potential marker for weight loss ${ }^{(4)}$. To keep individuals motivated, there is a need to focus on providing feedback over a 24-hour interval. Here, we aim to understand the use of ACE as an early marker for weight loss.

The participants followed a $1200 \mathrm{Kcal}$ diet with an optional daily $<250 \mathrm{Kcal}$-snack and used an in-house generated health platform to provide urine and diaries of food intake and physical activity ${ }^{(5)}$. The study was approved by Warwick Medical School Ethics committee BSREC. ACE concentration was measured using a Human ACE DuoSet Elisa. Pearson correlation and Mann Whitney U test were performed using $\mathrm{R}$ to understand correlation between ACE and weight change parameters.

A total of 47 individuals with mean BMI $27 \pm 5$ participated in the study. ACE levels after one day of dieting showed a linear trend in individuals with BMI $>25$ group (Figure 1A). Following a day of dieting, ACE positively correlated with weight difference significant at $p=0.015$ (Figure 1B). This reduction was more robust in individuals with a BMI $>25$ at $p=0.0015$ (Figure 1C). In the female participants, it was observed that the minimum ACE amount in a 24-hour interval was negatively correlated to weight lost $\left(\mathrm{R}^{2}=-0.453, \mathrm{p}=0.034\right.$; Figure 1D). Similarly, the following day ACE concentration was also positively correlated with weight difference $\left(R^{2}=0.482, p=0.031\right.$; Figure 1D).
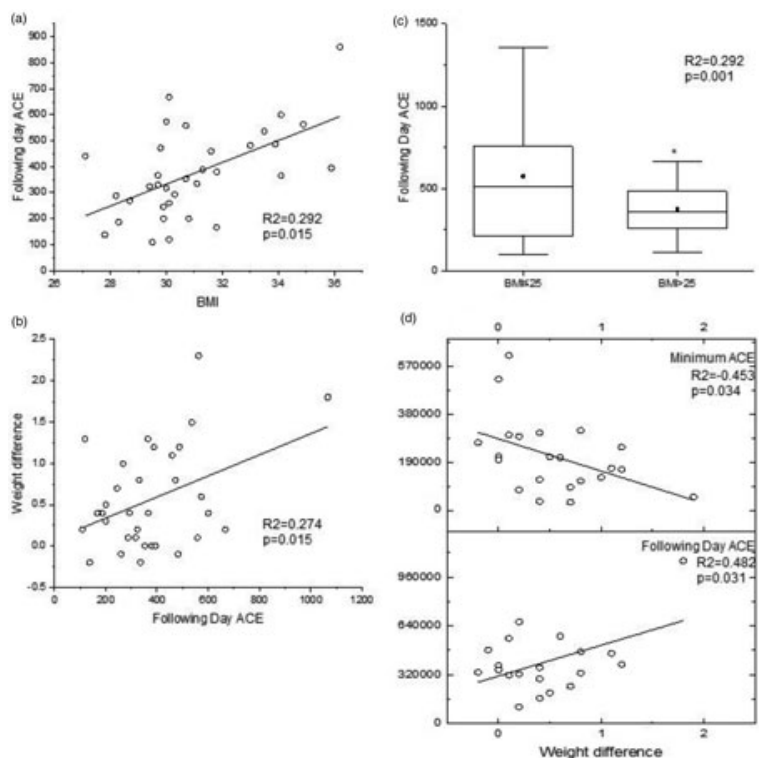

Fig. 1. ACE as an early biomarker for weight loss. A) Correlation of ACE with BMI; B) Correlation of ACE with weight difference; C) Comparison of following day ACE in BMI groups; D) Correlation of ACE with minimum and following day ACE in females.

Our study demonstrate that ACE levels vary within a 24-hour interval after following a caloric restricted diet and could thus potentially be used as an early biofeedback marker on dieting and weight loss. The response of ACE levels with weight loss and BMI after only 1 day of dieting could provide insights into personalised optimisation of dieting parameters.

1. Smith A \& Singleton (2013) J Diabetes Complicat 27, 436-442.

2. Schubert K, Alfano C, Duggan C et al. (2012) Obesity 20, 1628-1638.

3. Gong Z \& Gong Z (2012) Biology Direct 7, 19.

4. Wang P, Holst C, Andersen M, Astrup A et al. (2011) PLoS ONE 6, e16773.

5. Tejpal S, Bastie C \& Seetharaman J (2018) Proceedings of the Nutrition Society 77, E213. 